\title{
Economic anxiety among contingent survey workers
}

\author{
Meghan Condon ${ }^{1}\left[\right.$ ] Amber Wichowsky ${ }^{2}$
}

Accepted: 14 November 2021

(C) The Author(s), under exclusive licence to Springer Science+Business Media, LLC, part of Springer Nature 2021

\begin{abstract}
Psychologists and other social scientists increasingly conduct experiments with online convenience samples from Amazon's Mechanical Turk Marketplace (MTurk). MTurk and population-based samples differ in well-documented ways, but whether or not compositional differences are problematic for experiments remains controversial. We highlight a critically important characteristic that is likely to interact with many experimental treatments in the psychological and behavioral sciences, and that has not been identified by other studies of MTurk samples: economic anxiety. We document a sizable difference between contingent survey workers and the general population and explain the ways in which economic anxiety is likely to interact with experimental treatments. In an era of rapidly growing economic anxiety and group disparities in economic wellbeing, awareness of this compositional difference is essential, especially in cases where experimental stimuli may interact with economic anxiety.
\end{abstract}

Keywords Economic anxiety $\cdot$ MTurk $\cdot$ Online surveys $\cdot$ Experiments $\cdot$ Economic inequality

A great deal of experimental research in psychology and across the social sciences draws on convenience samples from Amazon's Mechanical Turk Marketplace (MTurk). MTurk workers tend to be younger, better educated, lower income, less racially and ethnically diverse, more liberal, and more politically interested than the general US population (Berinsky et al., 2012; Krupnikov \& Levine, 2014; Levay et al., 2016). These compositional differences are particularly important in studies of political psychology and group difference, where generalizability to the population is important.

We write this short report to draw attention to an important omission from the current set of known differences between MTurk and population-based samples: contingent survey workers display substantially higher levels of economic anxiety compared to the population. This difference is likely to produce effect heterogeneity in experiments in

Meghan Condon

mcondon1@luc.edu

1 Department of Political Science, Loyola University Chicago, 335 Coffey Hall 1032 W. Sheridan Road, Chicago, IL 60660, USA

2 Department of Political Science, Marquette University, Wehr Physics, Room 468, 1420 W. Clybourn St., Milwaukee, WI 53233, USA which treatments involve information processing, learning, persuasion, and social comparison. Attention to economic anxiety in such studies has only become more important during the COVID-19 pandemic with economic anxiety increasing dramatically and unevenly. COVID-19 related economic anxiety affects information seeking behaviors and is correlated with other consequential forms of anxiety (Vazirani and Bhattacharjee, 2020).

\section{Generalizability and MTurk Studies}

On balance, studies of MTurk worker representativeness recommend that researchers employ a series of covariates to increase generalizability. For example, Levay et al. (2016) recommend measuring age, gender, race and ethnicity, income, education, marital status, religion, ideology, and partisanship, using these variables to develop sample weights.

Other research suggests that the compositional differences between MTurk and national probability samples may be less concerning. Coppock (2019) demonstrates that several high-profile experiments of attitude change conducted with national probability samples replicate with online convenience samples, arguing that compositional differences in convenience samples are often unproblematic because 
many treatments produce similar effects across subjects. The important question then is not merely whether MTurk samples are unrepresentative of the population, but whether they are unrepresentative when it comes to characteristics likely to produce heterogeneous effects for a particular experiment.

Economic anxiety is clearly such a characteristic, in particular when it comes to political psychology. When people are anxious they become more wary of others, more sensitive to authoritarian messages and figures (Merolla \& Zechmeister, 2009), and more persuadable (Arceneaux, 2012). Anxious people are motivated to seek information, but their anxiety also biases the way they process that information (Brader, 2005, 2006; MacKuen et al., 2010; Valentino et al., 2008), with anxious individuals more likely to read, remember, and agree with threatening information (Albertson \& Gadarian, 2015; Brader, 2005, 2006; MacKuen et al., 2010; Valentino et al., 2008) This phenomenon is consistent with the high levels of political news interest among MTurk workers. Economic anxiety also affects opinion formation (Hacker et al., 2013; Margalit, 2013; Rehm et al., 2012), as well as social comparison and status perception (Condon \& Wichowsky 2020), and political engagement (Brooks, 2014; Levine, 2015).

\section{Economic Anxiety in MTurk Samples}

MTurk samples draw from a pool of Americans we might expect to be exceptionally anxious about their economic circumstances. While there is heterogeneity in the pool of on-demand workers, a sizable share do gigs because of economic need: because they need the supplemental income or because more stable jobs are unavailable (Manyika et al., 2016). On-demand workers are only paid for time they spend working, which can vary a great deal across weeks and months (GAO, 2015). These workers therefore experience more income volatility than salaried workers. Contingent workers are not covered by minimum wage or overtime compensation laws, enjoy fewer legal protections than workers classified as employees, and are much less likely to receive workplace benefits.

We measure economic anxiety in an MTurk sample and compare it to the same measures administered in a nationally representative probability sample from the Marketplace Economic Anxiety Index. MTurkers in our study were 18 years or older and from the U.S. We direct readers to the supplemental appendix (Table A3), which provides the descriptive statistics for our MTurk sample (age, gender, region, education, income, race/ethnicity) and shows how our sample compares to U.S. Census benchmarks (18 year and older population). Consistent with other research on the composition of MTurkers, our sample is younger, less racially diverse, and more likely to be college educated. The median household income of our sample is close to the U.S. median household income and the geographic composition also mirrors that of the United States. The unemployment rate among our study participants was double (8.1 percent) that of the national unemployment rate (4 percent) at the time of our study. ${ }^{1}$ We find that MTurk workers express much higher levels of economic anxiety. Adjusting for the demographic characteristics of the sample decreases, but does not wash away the anxiety gap.

The Marketplace Edison Research Survey is a nationally representative survey of Americans 18 and older. ${ }^{2}$ Survey Sampling International (SSI) provided the phone numbers and email addresses for the telephone and online survey. Just over one thousand respondents participated in the April 2017 Marketplace survey $(\mathrm{N}=1,044)$, with roughly equal numbers of interviews conducted by telephone and online. Our MTurk survey was conducted in October 2017 $(\mathrm{N}=200)$. Economic conditions had improved slightly: the unemployment rate had dropped from 4.4 to 4.1, and the stock market had also experienced gains. These improvements, if anything, would bias us against finding higher levels of anxiety in the MTurk sample.

We benchmarked our MTurk sample to the Marketplace Economic Anxiety Index using two questions. The questions asked respondents how often they feel anxious about their financial situation (never, rarely, often, frequently) and how secure they feel about their financial situation (financially secure, somewhat financially secure, not financially secure, or don't know). Both items are scaled such that higher values reflect greater insecurity. For means and standard errors, see Table A1 in the supplemental appendix. We first consider the raw data. On average, subjects in our MTurk sample reported feeling more anxious $(\Delta=0.30, \mathrm{SE}=0.07, \mathrm{t}=4.09$, $\mathrm{p}<0.001)$ and insecure $(\Delta=0.39, \mathrm{SE}=0.05, \mathrm{t}=7.70$, $\mathrm{p}<0.001)$ than Marketplace survey respondents. These differences between the surveys are sizable; comparing to the nationally representative sample, they translate into nearly a third of a standard deviation on the anxiety scale and about one-half of a standard deviation on the insecurity scale. The differences are also larger than the gap between subjects with and without college degrees in the MTurk sample.

When we drill down to more specific economic concerns, we largely see the same pattern. For example, MTurk

\footnotetext{
1 This study included a check for attention. After the economic anxiety study reported here, we used the same MTurk sample to pilot an intervention for a separate experiment. This second study included a brief open-ended response item asking a randomly selected half of the sample to read and reflect in writing on a vignette. One-hundred percent of these subjects wrote something relevant in response to the open-ended item.

${ }^{2}$ Surveys can be found at https://www.marketplace.org/topics/econo my/anxiety-index.
} 
workers are more concerned about saving enough to pay for retirement. (See Table A2 in the supplemental appendix.) This difference could be driven by the absence of employerprovided benefits among MTurkers, or by age, with younger MTurk workers farther away from savings goals.

The Marketplace survey is a national probability sample, weighted to match the most recent population estimates from the U.S. Census Bureau for age, gender, race, income and region of the country. When we use the same covariates to weight our MTurk sample, the sample differences in anxiety $(\Delta=0.23, \mathrm{SE}=0.08, \mathrm{t}=2.91, \mathrm{p}<0.01)$ and insecurity $(\Delta=0.38, \mathrm{SE}=0.06, \mathrm{t}=6.90, \mathrm{p}<0.001)$ shrink only slightly and remain statistically significant.

\section{Discussion}

This finding has important implications across the psychological and behavioral sciences in this new era of economic anxiety and inequality. The anxiety difference should be especially concerning for scholars employing experimental interventions that may interact with subjects' levels of economic anxiety or insecurity. Several other studies have demonstrated that experimental stimuli involving authoritarian messages, information processing, learning, persuasion, social comparison, and political engagement interact with anxiety. Scholars in these fields should carefully consider the implications of using a particularly anxious experimental sample.

There is recent evidence that experiments conducted with national probability samples often do replicate with MTurk samples. This evidence has come primarily from studies of opinion change (Coppock, 2019). Researchers should be cautious in extending this conclusion to studies of other phenomena, in particular in studies that investigate economic issues and the politics of economic inequality.

Our purpose, in this research note, has been to describe and document this important compositional difference between contingent worker and general population samples. Future research can follow inquiry on opinion change and examine the replicability of specific interventions in MTurk and national probability samples. On the other hand, MTurk is fruitful ground for recruiting subjects who are especially economically anxious, if the goals of a study require it. More broadly, this finding underscores the simple fact that important unmeasured differences between convenience and probability samples are likely to remain, even after adjusting for the growing list of characteristics known to vary between them.

Supplementary Information The online version contains supplementary material available at https://doi.org/10.1007/s12144-021-02535-4.
Funding This study was funded by Marquette University.

Data Availability The Marketplace Edison Research Surveys can be found at https://www.marketplace.org/topics/economy/anxiety-index. Data collected by the authors will be made available on author website upon publication.

Code Availability Available upon request.

\section{Declarations}

Conflicts of Interest/Competing Interests The authors have no conflicts of interest to declare that are relevant to the content of this article.

Consent to Participate This study received approval from the Marquette University Human Subjects Committee under IRB protocol no. HR-2760.

\section{References}

Albertson, B., \& Gadarian, S. K. (2015). Anxious politics: Democratic citizenship in a threatening world. Cambridge University Press.

Arceneaux, K. (2012). Cognitive biases and the strength of political arguments. American Journal of Political Science, 56(2), 271-285.

Berinsky, A. J., Huber, G. A., \& Lenz, G. S. (2012). Evaluating online labor markets for experimental research: Amazon. com's Mechanical Turk. Political Analysis, 20(3), 351-368.

Brader, T. (2005). Striking a responsive chord: How political ads motivate and persuade voters by appealing to emotions. American Journal of Political Science, 49(2), 388-405.

Brader, T. (2006). Campaigning for hearts and minds: How emotional appeals in political ads work. University of Chicago Press.

Brooks, S. M. (2014). Insecure democracy: Risk and political participation in Brazil. The Journal of Politics, 76(4), 972-985.

Coppock, A. (2019). Generalizing from survey experiments conducted on Mechanical Turk: A replication approach. Political Science Research and Methods, 7(3), 613-628.

GAO. (2015). Contingent Wo\#\#rkforce: Size, characteristics, earnings, and benefits. (GAO-15-168R). Washington, DC

Hacker, J. S., Rehm, P., \& Schlesinger, M. (2013). The insecure American: Economic experiences, financial worries, and policy attitudes. Perspectives on Politics, 23-49.

Krupnikov, Y., \& Levine, A. S. (2014). Cross-sample comparisons and external validity. Journal of Experimental Political Science, $1(1), 59-80$

Levay, K. E., Freese, J., \& Druckman, J. N. (2016). The demographic and political composition of Mechanical Turk samples. SAGE Open, 6(1). https://doi.org/10.1177/2158244016636433

Levine, A. S. (2015). American insecurity: Why our economic fears lead to political inaction: Princeton University Press.

MacKuen, M., Wolak, J., Keele, L., \& Marcus, G. E. (2010). Civic engagements: Resolute partisanship or reflective deliberation. American Journal of Political Science, 54(2), 440-458.

Manyika, J., Lund, S., Bughin, J., Robinson, K., Mischke, J., \& Mahajan, D. (2016). Independent work: Choice, necessity, and the gig economy. McKinsey Global Institute. In: October.

Margalit, Y. (2013). Explaining social policy preferences: Evidence from the Great Recession. American Political Science Review, 107(01), 80-103. 
Merolla, J. L., \& Zechmeister, E. J. (2009). Terrorist threat, leadership, and the vote: Evidence from three experiments. Political Behavior, 31(4), 575.

Rehm, P., Hacker, J. S., \& Schlesinger, M. (2012). Insecure alliances: Risk, inequality, and support for the welfare state. American Political Science Review, 106(02), 386-406.

Valentino, N. A., Hutchings, V. L., Banks, A. J., \& Davis, A. K. (2008). Is a worried citizen a good citizen? Emotions, political information seeking, and learning via the internet. Political Psychology, 29(2), 247-273.
Vazirani, A., \& Bhattacharjee, T. (2020). COVID-19 lockdowns and a response model to health, economic and food anxiety. Current Psychology, 1-11.

Publisher's Note Springer Nature remains neutral with regard to jurisdictional claims in published maps and institutional affiliations. 